\title{
Impact of an educational intervention and clinical performance dashboard on neonatal bloodstream infections
}

\author{
M S Raban, ${ }^{1}$ FCPaed (SA), MPhil Neonatol, Cert Neonatol (SA); C Bamford, ${ }^{2}$ MB ChB, DCH (SA), MPhil, FCPath (Micro), \\ MMed (Med Micro); Y Joolay, ${ }^{1}$ FCPaed (SA), MPhil Neonatol, Cert Neonatol (SA); M C Harrison, ${ }^{1}$ MRCP, FRCPCH \\ ${ }^{1}$ Division of Neonatal Medicine, Department of Paediatrics, Faculty of Health Sciences, University of Cape Town, South Africa \\ ${ }^{2}$ National Health Laboratory Service, Groote Schuur Hospital and Division of Medical Microbiology, Department of Clinical Laboratory Sciences, \\ Faculty of Health Sciences, University of Cape Town, South Africa
}

Corresponding author: M S Raban (shukriraban@yahoo.co.uk)

Background. Blood cultures are the most direct method of detecting bacteraemia. Reducing contamination rates improves the specificity and positive predictive value of the blood culture. Clinical performance dashboards have been shown to be powerful tools in improving patient care and outcomes.

Objectives. To determine whether prospective surveillance of bloodstream infections (BSIs), introduction of an educational intervention and the use of a clinical performance dashboard could reduce BSIs and blood culture contamination rates in a neonatal nursery.

Methods. We compared two time periods, before and after an intervention. Blood culture data were extracted from the local microbiology laboratory database. The educational intervention included the establishment of hand-washing protocols, blood culture techniques and video tools. A clinical performance dashboard was developed to demonstrate the monthly positive blood culture and contamination rates, and this was highlighted and referred to weekly at the unit staff meeting.

Results. Before the intervention, 1460 blood cultures were taken; 206 (14.1\%) were positive, of which 104 (7.1\% of the total) were contaminants. In the period following the intervention, 1282 blood cultures were taken; 131 (10.2\%) were positive, of which 42 (3.3\% of the total) were contaminants. The number of positive blood cultures and contamination rates after the intervention were both statistically significantly reduced ( $p=0.002$ and $p<0.001$, respectively).

Conclusion. This study demonstrates that adopting a relatively simple educational tool, making use of a clinical performance dashboard indicator and benchmarking practice can significantly reduce the level of neonatal sepsis while also reducing contaminated blood cultures.

S Afr Med J 2015;105(7):564-566. DOI:10.7196/SAMJnew.7764

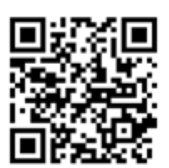

Advances in neonatal intensive care have improved survival of preterm infants. ${ }^{[1]}$ Despite these advances, neonatal infection remains an important cause of mortality, morbidity and prolonged hospital stays. ${ }^{[2,3]}$

Blood cultures are the most direct method of detecting bacteraemia in patients. ${ }^{[4]}$ Contaminated blood culture results can be troublesome for clinicians attempting to determine whether the organism represents a significant infection requiring intervention or is merely a false-positive result with no consequence to the patient. Reducing contamination rates will improve the specificity of the blood culture and result in a higher positive predictive value, resulting in a significantly more useful test. ${ }^{[5]}$

To guide empirical antibiotic treatment, infection surveillance is important to monitor infection rates, patterns of sensitivity profiles and infection control measures. It can also be used for benchmarking practice and for interventional studies. ${ }^{[6]}$

Clinical performance dashboards that present and benchmark performance against a series of key indicators have been shown to be powerful tools in improving patient care and outcomes. ${ }^{[7]}$

We evaluated the impact of prospective surveillance of bloodstream infections (BSIs), the introduction of an educational intervention and the use of a clinical performance dashboard on the total number of BSIs and blood culture contamination rates.

\section{Methods}

The study was conducted at the tertiary-level neonatal unit at Groote Schuur Hospital (GSH), Cape Town, South Africa (SA), over a 31-month period. The study was approved by the Human Research Ethics Committee, Faculty of Health Sciences, University of Cape Town.

The total number of blood cultures performed and the number of cultures yielding growth were extracted from the National Health Laboratory Service microbiology laboratory database on a monthly basis. BSI was defined as a positive blood culture including fungal organisms. If multiple blood cultures were taken within a 48-hour period and contained the same organism, we considered this a single BSI.

A positive blood culture was considered to be a contaminant if any of the following organisms was detected: alpha-haemolytic streptococci, Micrococcus spp., Propionibacterium spp., Corynebacterium spp., Bacillus spp., and sometimes enterococci and Acinetobacter spp., depending on the clinical status of the patient. ${ }^{[5]}$ In cases of coagulasenegative Staphylococcus-positive blood cultures, the cultures were classified as contaminants if serial serum C-reactive protein levels measured 24 hours apart were $<10 \mathrm{mg} / \mathrm{L}$, if repeated blood cultures remained negative, or if the attending clinician did not initiate antibiotic treatment because the infant did not have clinical features of sepsis. ${ }^{[8]}$

We examined and compared two time periods, period 1 (January 2010 - April 2011) before any intervention, and period 2 (May 2011 - 
July 2012) following the introduction of an educational intervention and use of a clinical performance dashboard.

The intervention included written policies prioritising and re-enforcing hand washing and a 'bare-below-elbows' approach, establishing uniform blood culture technique, appropriate antibiotic use, and insertion and management of central lines according to a standardised protocol. Video material was used to demonstrate the techniques described in the above protocols. A clinical performance dashboard was developed as a visual aid to demonstrate the monthly positive blood culture and contamination rates, and this was highlighted and referred to weekly at the neonatal unit staff meeting. We calculated a baseline median for the number of BSIs in the unit for the period January - December 2010. The median was calculated annually and only adjusted if it was less than that in the previous year. This 'rolling' median served as the benchmark against which performance was monitored. The clinical performance dashboard provided the nursing and medical staff in the neonatal unit with a real-time, instantaneous picture of their performance with regard to the total number of positive blood cultures and the contamination rates. These data were visually displayed using a colour-coded system: green, when the total number of positive blood cultures was less than the median; blue, when the total number of positive blood cultures equalled the median; and red, when the total number of positive blood cultures breached the median. Fig. 1 illustrates how the dashboard was utilised in the neonatal unit.

\section{Data analysis}

The microbiology laboratory data were exported to a Microsoft Excel file. Data were analysed with Stata version 12 (Stata Corporation, USA). Chi-square and Fisher's exact tests were used for comparison of categorical variables. Descriptive results are expressed as numbers and proportions (\%). A $p$-value of $<0.05$ was considered significant.

\section{Results}

During period 1, 1460 blood cultures were taken; 206 (14.1\%) were positive, of which 104 (7.1\% of the total) were contaminants. During period 2, following the introduction of an educational intervention and use of a clinical performance dashboard, 1282 blood cultures were taken; 131 (10.2\%) were positive, of which 42 (3.3\% of the total) were contaminants. There were statistically significant reductions in BSIs $(p=0.002)$ and

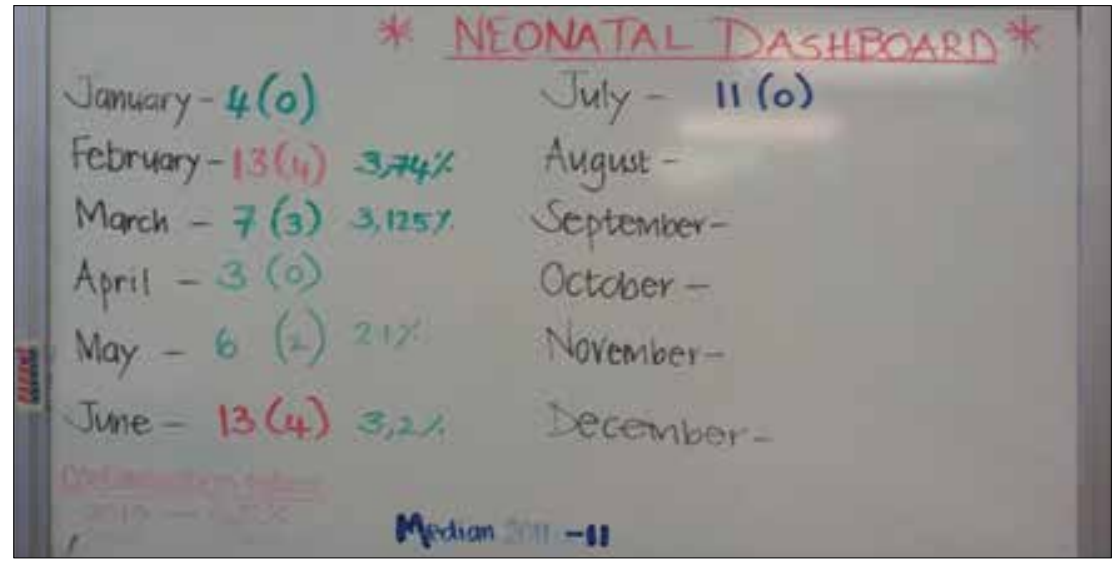

Fig. 1. The performance dashboard, a visual indicator tool displayed in the neonatal unit at Groote Schuur Hospital. Values written in green indicate months in which positive cultures were below the median number of positive cultures. Red values are used to indicate months when positive cultures were above the median benchmark.

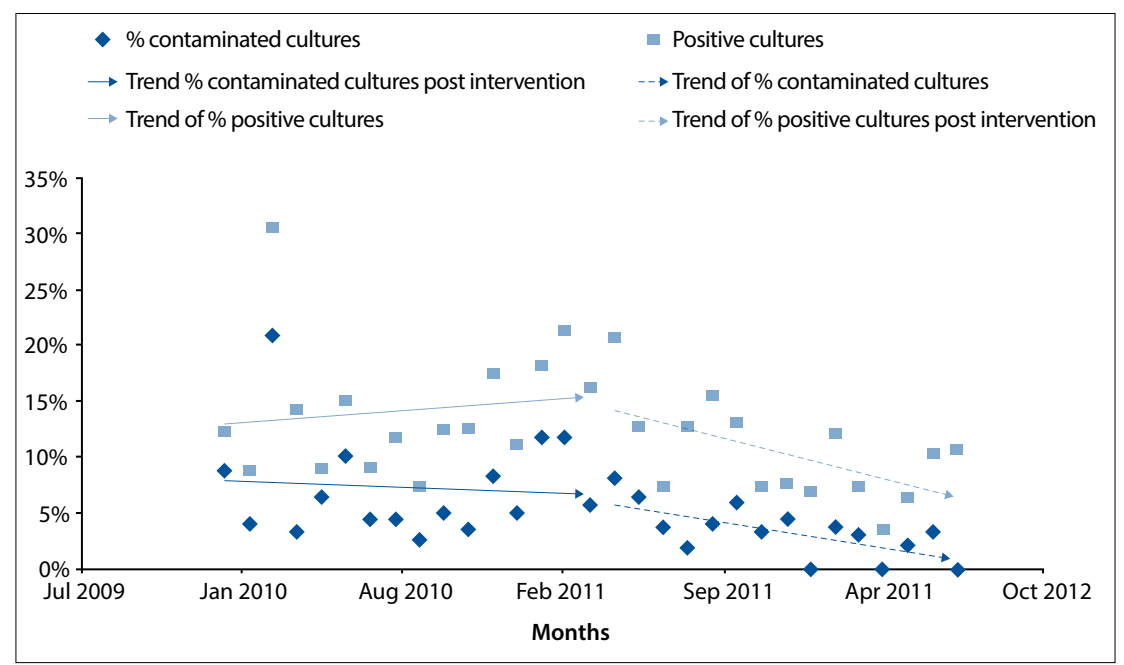

Fig. 2. Percentages of positive cultures and contaminants before and after intervention.

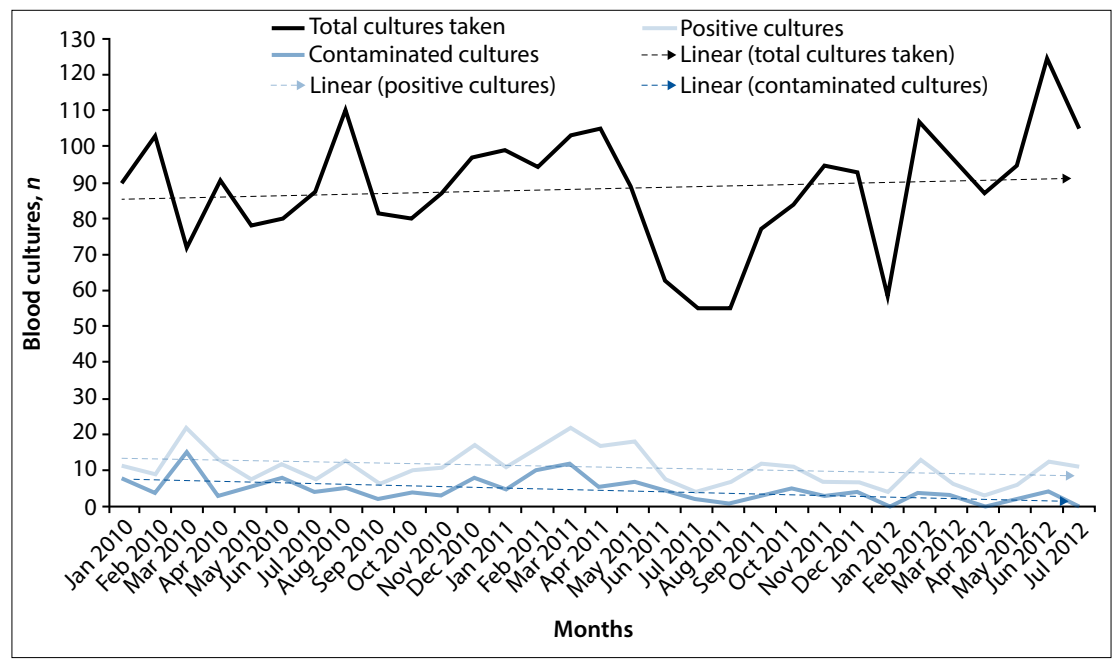

Fig. 3. Monthly trend of total, positive and contaminated blood cultures.

blood culture contamination rates $(p<0.001)$ between the pre- and post-intervention periods. Fig. 2 illustrates the decline in BSIs and blood culture contamination rates following the intervention. While the total number of blood cultures taken in the neonatal unit fluctuated from month to month, over the study period the 
general trend between period 1 and period 2 remained unchanged. Importantly, the admission rates and referral patterns remained unchanged during the two study periods, suggesting that the workload and clinical indications for taking the blood culture were similar for period 1 and period 2. Fig. 3 illustrates the relationship between the total number of blood cultures taken, BSIs and contaminated blood cultures.

\section{Discussion}

Improving the quality and safety of healthcare services is of paramount importance. Surveillance and monitoring of key indicators is central in achieving this, as it allows for benchmarking and performance comparison. Even more important is identifying and implementing interventions that impact significantly on these key indicators. A rapidly growing body of evidence suggests that quality improvement strategies in a variety of clinical settings, including neonatal care, are effective and improve patient outcomes while also reducing costs. ${ }^{[6]}$

Our study demonstrates a significant measurable reduction in the total number of neonatal BSIs and blood culture contamination rates. This is an important outcome, as Modi and Carr ${ }^{[9]}$ and Stoll et al. ${ }^{[10]}$ documented neonatal BSI rates for all neonates of $10-25 \%$ and rates as high as $50 \%$ in extremely preterm infants. In a recent meta-analysis, Bakhuizen et al.$^{[11]}$ concluded that neonatal BSI is associated with an increased risk of mortality and severe short- and long-term outcomes.

Contaminated blood cultures have a significant impact on the patient and, also importantly, on cost. Compared with truly negative cultures, false-positive cultures increase laboratory workload, prolong patient stay and increase the use of broad-spectrum antibiotics, with negative consequences for antibiotic resistance and patient morbidity. ${ }^{[12]}$ The American Society for Microbiology suggests contamination rates of $<3 \%$ for all blood cultures taken. ${ }^{[5]}$ We reduced the blood culture contamination rate from $7 \%$ to $3.3 \%$. Hand washing is recognised as the single most effective method of reducing the transmission of micro-organisms between patients, and is an integral part of hospital infection control measures. ${ }^{[13]}$ Blood culture contamination is a result of poor techniques of hand washing, skin cleaning and venepuncture. ${ }^{[12]}$

An essential part of our stratagem of reducing BSI and contamination was the clinical performance dashboard. The high visual impact of the dashboard permitted nursing and medical staff to benchmark their performance against the set threshold and helped drive performance excellence. The dashboard also served as an excellent communication tool; night staff in particular benefited from this, as they were often unable to attend the neonatal unit staff meetings during normal working hours. The dashboard provided the unit with real-time data allowing us to anticipate and recognise problems earlier (e.g. when BSI results equalled the median or breached the median) and consequently to rectify these problems more timeously.

\section{Study limitations}

This study does have limitations. It did not quantify the impact of the intervention on hospital resource utilisation, particularly length of hospital stay, antibiotic utilisation and cost-effectiveness. In addition, it did not explore the impact that reduction of BSIs and blood culture contamination rates had on mortality and short- and long-term morbidities.

\section{Conclusion}

This study demonstrates that adopting a relatively simple educational tool, utilisation of a clinical performance dashboard indicator and benchmarking practice can significantly reduce the level of neonatal sepsis while also reducing contaminated blood cultures. This stratagem was based on intensive collaboration with, and among, all levels of staff over the study period and continues to prove successful in our endeavours to reduce infection rates in the GSH neonatal unit. Given the impact of neonatal BSIs on patient outcomes and hospital resource utilisation, the establishment of local and national neonatal infection surveillance networks, with similar interventions to those we report, is imperative to monitor the epidemiology of neonatal infections in SA and inform policy and clinical practice. Integrating and leveraging quality improvement benchmarks derived from surveillance and monitoring into clinical performance dashboards can drive initiatives aimed at excellence within all spheres of healthcare services, at both local and national levels.

\section{References}

1. Hack M, Fanaroff AA. Outcomes of children of extremely low birthweight and gestational age in the 1990s. Semin Neonatol 2000;5(2):89-106. [http://dx.doi.org/10.1053/siny.1999.0001]

2. Modi N, Doré CJ. Saraswatula A, et al A case definition for national and international neonatal Modi N, Doré CJ, Saraswatula A, et al. A case definition for national and international neonatal
bloodstream infection surveillance. Arch Dis Child Fetal Neonatal Ed 2009;94(1):F8-F12. [http:// bloodstream infection surveillance.

dx.doi.org/10.1136/adc.2007.126458
Adams-Chapman I, Stoll BJ. Neonatal infection and long-term neurodevelopmental outcome in the preterm infant. Curr Opin Infect Dis 2006;19(3):290-297. [http://dx.doi.org/10.1097/01 qco.0000224825.57976.87]

4. Ruge DG, Sandin RL, Siegelski S, et al. Reduction in blood culture contamination rates by establishmen of policy for central intravenous catheters. Lab Med 2002;33(10):797-800.

Hall KK, Lyman JA. Updated review of blood culture contamination. Clin Microbiol Rev 2006;19(4):788-802. [http://dx.doi.org/10.1128/CMR.00062-05]

Horbar JD, Rogowski J, Plsek PE, et al. Collaborative quality improvement for neonatal intensive care. Pediatrics 2001;107(1):14-22. [http://dx.doi.org/10.1542/peds.107.1.14]

Egan M. Clinical dashboards impact on workflow, care quality, and patient safety. Crit Care Nurs $Q$ 2006;29(4):354-361

8. Weinbaum FI, Lavie S, Danek M, Sixsmith D, Heinrich GF, Mills SS. Doing it right the first time: Quality improvement and the contaminant blood culture. J Clin Microbiol 1997;35(3):563-565.

Modi N, Carr R. Promising stratagems for reducing the burden of neonatal sepsis. Arch Dis Child Fetal Modi N, Carr R. Promising stratagems for reducing the burden of neonatal sep
Neonatal Ed 2000;83(2):F150-F153. [http://dx.doi.org/10.1136/fn.83.2.F150]

10. Stoll BJ, Hansen N, Fanaroff AA, et al. Late-onset sepsis in very low birth weight neonates: The Stoll BJ, Hansen N, Fanaroff AA, et al. Late-onset sepsis in very low birth weight neonates: The
experience of the NICHD Neonatal Research Network. Pediatrics 2002;110(2):285-291. [http://dx.doi. org/10.1542/peds.110.2.285

11. Bakhuizen SE, de Haan TR, Teune MJ, et al. Meta-analysis shows that infants who have suffered neonatal sepsis face an increased risk of mortality and severe complications. Acta Paediat 2014;103(12):1211-1218. [http://dx.doi.org/10.1111/apa.12764]

12. Roth A, Wiklund AE, Pålsson AS, et al. Reducing blood culture contamination by a simple informational intervention. J Clin Microbiol 2010;48(12):4552-4558. [http://dx.doi.org/10.1128/ JCM.00877-10]

13. Larson E. Skin hygiene and infection prevention: More of the same or different approaches? Clin Infect Dis 1999;29(5):1287-1294. [http://dx.doi.org/10.1086/313468]

Accepted 15 September 2014. 